\title{
Information content versus word length in random typing
}

\author{
Ramon Ferrer-i-Cancho ${ }^{1, *}$ and Fermín Moscoso del Prado \\ Martín ${ }^{2,3,4}$ \\ ${ }^{1}$ Complexity \& Quantitative Linguistics Lab \\ Departament de Llenguatges i Sistemes Informàtics, \\ TALP Research Center, Universitat Politècnica de Catalunya, \\ Campus Nord, Edifici Omega Jordi Girona Salgado 1-3. \\ 08034 Barcelona, Catalonia (Spain) \\ ${ }^{2}$ Laboratoire de Psychologie Cognitive (UMR6146) \\ CNRS \& Aix-Marseille Université I, Marseille, France \\ ${ }^{3}$ Laboratoire Dynamique du Langage (UMR5596) \\ CNRS \& Université de Lyon II, Lyon, France \\ ${ }^{4}$ Institut Rhône-Alpin de Systèmes Complexes, Lyon, France \\ E-mail: rferrericancho@lsi.upc.edu and fermin.moscoso-del-prado@gmail.com
}

\begin{abstract}
Recently, it has been claimed that a linear relationship between a measure of information content and word length is expected from word length optimization and it has been shown that this linearity is supported by a strong correlation between information content and word length in many languages (Piantadosi et al. 2011, PNAS 108, 3825-3826). Here, we study in detail some connections between this measure and standard information theory. The relationship between the measure and word length is studied for the popular random typing process where a text is constructed by pressing keys at random from a keyboard containing letters and a space behaving as a word delimiter. Although this random process does not optimize word lengths according to information content, it exhibits a linear relationship between information content and word length. The exact slope and intercept are presented for three major variants of the random typing process. A strong correlation between information content and word length can simply arise from the units making a word (e.g., letters) and not necessarily from the interplay between a word and its context as proposed by Piantadosi et al. In itself, the linear relation does not entail the results of any optimization process.
\end{abstract}

Keywords: Zipf's law of brevity, random typing, uniform information density.

PACS numbers: 89.70.-a Information and communication theory

89.75.Da Systems obeying scaling laws

05.40.-a Fluctuation phenomena, random processes, noise, and Brownian motion

02.30.Lt Sequences, series, and summability

Submitted to: Journal of Statistical Mechanics: theory and experiment (JSTAT) 


\section{Introduction}

In his pioneering research, G. K. Zipf showed that more frequent words tend to be shorter [1], and parallels of this brevity law have been reported for the behavior of other species [2, 3]. Recently, it has been argued that "average information content is a much better predictor of word length than frequency" and that this "indicates that human lexicons are efficiently structured for communication by taking into account interword statistical dependencies." [4, p. 1]. According to the uniform information density hypothesis (e.g., [5]), "language users make choices that keep the number of bits of information communicated per unit of time approximately constant" and thus "the amount of information conveyed by a word should be linearly related to the amount of time it takes to produce -approximately, its length- to convey the same amount of information in each unit of time" [4, p. 1]. Here it will be shown that hitting keys from a keyboard at random (e.g., [6, 7]) generates words that reproduce this linear relationship. Therefore, the observation of such a linear relationship does not constitute unequivocal evidence for any kind of optimal choices made by speakers.

Throughout this paper, $C$ denotes contexts and $W$ denotes words. As in Ref. [4, the context of a word consists of a fixed number of preceding words, and the information content of a word $w$ is given by

$$
I(w)=-\sum_{c} p(C=c \mid W=w) \ln p(W=w \mid C=c) .
$$

The expected information content of words of length $\ell$ is defined as [4]

$$
I(\ell)=\sum_{\|w\|=\ell} p(W=w \mid\|w\|=\ell) I(w),
$$

where $\|w\|$ is the length (in letters) of a word $w$ and $\ell$ is a fixed parameter value. In this study, we detail some connections between $I(w)$ and standard information theory measures. The definition of $I(w)$ that we borrow from Ref. [4] is somewhat idiosyncratic in relation to standard information-theory. We found that, Ref. [8], the reference supplied in Ref. 4] as a justification for Eq. 1, does not in fact justify the equation in any evident way. In this study we demonstrate that $I(\ell)$ is a linear function of $\ell$ for a general class of random typing processes. The only requirement is that the context is defined by means of neighbouring words (as in [4]) or that empty words (words of length zero) are allowed as in many variants of the random typing process [6, 9, 10].

\section{Connections with standard information theory}

We now introduce our basic notation and conventions. The self-information of an event that has probability $p$ is $-\ln p$. We consider $C$ and $W$ independent if and only if $p(C=c, W=w)=p(C=c) p(W=w)$. As usual, by the definition of conditional probability, independence implies both $p(C=c \mid W=w)=p(C=c)$ and $p(W=w \mid C=c)=p(W=w)$, for any individual $c$ and $w$. Therefore, under independence between $C$ and $W$, it holds that $I(w)=I_{0}(w)=-\ln p(W=w)$, that is 
to say, $I(w)$ is just the self-information of $w$. The expected self-information content of a word of length $\ell$ is

$$
\begin{aligned}
I_{0}(\ell) & =-\sum_{\|w\|=\ell} p(W=w \mid\|w\|=\ell) \ln p(W=w) \\
& =-\sum_{\|w\|=\ell} p(W=w \mid\|w\|=\ell) \ln p(W=w,\|w\|=\ell) .
\end{aligned}
$$

In sum, under independence between $C$ and $W, I(\ell)$ and $I_{0}(\ell)$ coincide.

The conditional entropy is defined as,

$$
\begin{aligned}
H(W \mid C) & =\sum_{c} p(C=c) H(W \mid C=c) \\
& =-\sum_{c} p(C=c) \sum_{w} p(W=w \mid C=c) \ln p(W=w \mid C=c) .
\end{aligned}
$$

Given only the joint probability, i.e. $p(W=w, C=c)$, one can use Bayes' Theorem for calculating the conditional and marginal probabilities, as it was done in previous work [4] and is assumed by various information theoretic models of Zipf's law for word frequencies [11, 12]. Simple application of Bayes' Theorem to the definition of $H(W \mid C)$ in (3) shows that the conditional entropy is the expectation of $I(w)$ :

$$
\begin{aligned}
H(W \mid C) & =-\sum_{c} \sum_{w} p(W=w, C=c) \ln p(W=w \mid C=c) \\
& =-\sum_{w} p(W=w) \sum_{c} \frac{p(W=w, C=c)}{p(W=w)} \ln p(W=w \mid C=c) \\
& =-\sum_{w} p(W=w) \sum_{c} p(C=c \mid W=w) \ln p(W=w \mid C=c) \\
& =\sum_{w} p(W=w) I(w)=E[I(w)] .
\end{aligned}
$$

It is not difficult to see that $I_{0}(w)$ is the upper bound of $I(w)$ and $H(C \mid w)$ is its lower bound; formally,

$$
H(C \mid w) \leq I(w) \leq I_{0}(w) .
$$

As for a lower bound of $I(w)$, the relative entropy (or Kullback-Leibler divergence) between the context conditional probability and the word conditional probability is [13]

$$
\begin{aligned}
D(p(C=c \mid W=w) \| p(W=w \mid C=c))= & \sum_{c} p(C=c \mid W=w) \ln \frac{p(C=c \mid W=w)}{p(W=w \mid C=c)} \\
= & \sum_{c} p(C=c \mid W=w) \ln p(C=c \mid W=w) \\
& -\sum_{c} p(C=c \mid W=w) \ln p(W=w \mid C=c) \\
= & I(w)-H(C \mid w) .
\end{aligned}
$$

Therefore $I(w) \geq H(C \mid w)$ by the non-negativity of the relative entropy [13]. As for the upper bound of $I(w)$, the non-negativity of mutual information, i.e. $I(W ; C)=$ $H(W)-H(W \mid C) \geq 0[13$ and (4), yields

$$
H(W \mid C) \quad \leq H(W)
$$


Information content versus word length in random typing

$$
\begin{aligned}
\sum_{w} p(W=w) I(w) & \leq-\sum_{w} p(W=w) \ln p(W=w) \\
& =\sum_{w} p(W=w) I_{0}(w)
\end{aligned}
$$

if and only if $I(w) \leq I_{0}(w)$, as we wanted to prove. Combining (11) and (5) results in

$$
I_{C}(\ell) \leq I(\ell) \leq I_{0}(\ell),
$$

where $I_{C}(\ell)$ is defined as

$$
I_{C}(\ell)=\sum_{\|w\|=\ell} p(W=w \mid\|w\|=\ell) H(C \mid w) .
$$

\section{Information content versus length in random typing}

Random typing [6, 10] is a process in which a sequence of characters is produced by sampling randomly from a set of possible characters. Here we consider a generalized random typing model based upon variants allowing for unequal letter probabilities as in [7, 10] and allowing one to specify a minimum word length [14].

Assume that characters are produced from an alphabet $\Sigma=\left\{\sigma_{0}, \ldots, \sigma_{i}, \ldots, \sigma_{\lambda-1}\right\}$, where $\lambda$ is the alphabet size, $\sigma_{0}$ represents the word delimiter (i.e., the space character) and the remaining characters of $\Sigma$ are letters. We assume that all the characters in $\Sigma$ are produced at random and independently, with the only exception that two instances of the space character must be separated by at least $\ell_{0}$ intervening characters other than the space. In such model, the production of a word is separated into two phases: generation of the space-free prefix of length $\ell_{0}$, and generation of the remainder. $S$ is a random variable taking values from $\Sigma$ as generated by the random typing process. $p_{\Sigma}(S=s)$ is defined as the probability of producing character $s$ as the $k$-th character after the last space produced (or after the beginning of the sequence if no space has been produced yet), for any value $k \geq \ell_{0} \cdot p_{\Sigma \backslash\left\{\sigma_{0}\right\}}(S=s)$ is the same probability as $p_{\Sigma}(S=s)$ for values of $k<\ell_{0}$. The abbreviation $p_{0}=p_{\Sigma}\left(S=\sigma_{0}\right)$ will be used hereafter. We assume that $p_{\Sigma}(S=s)>0$ for all characters in $\Sigma$ with the additional constraint that $p_{0}<1 . p_{\Sigma \backslash\left\{\sigma_{0}\right\}}(S=s)$ is defined in terms of $p_{\Sigma}(S=s)$,

$$
p_{\Sigma \backslash\left\{\sigma_{0}\right\}}(S=s)=\left\{\begin{array}{cc}
\frac{p_{\Sigma}(S=s)}{1-p_{0}} & \text { if } s \neq \sigma_{0} \\
0 & \text { if } s=\sigma_{0} .
\end{array}\right.
$$

The generalized random typing process with unequal letter probabilities is defined by $\lambda$ parameters: $\ell_{0}$ and the $\lambda-1$ probabilities $p_{\Sigma}\left(S=\sigma_{i}\right)$ for $0 \leq i \leq \lambda-2$ with

$$
p_{\Sigma}\left(S=\sigma_{\lambda-1}\right)=1-\sum_{i=0}^{\lambda-2} p_{\Sigma}\left(S=\sigma_{i}\right) .
$$

Notice the additional parameter $\ell_{0}$ that is not considered in other versions of the random typing model and allows for unequal character probabilities [7, 10].

In the remainder of this section we start by proving that $I_{0}(\ell)$ is a linear function of $\ell$, providing exact analytical expressions for its slope and intercept. We continue by showing that $I(\ell)$ can be inferred from $I_{0}(\ell)$. If the context is defined by words, as in 
Ref. [4], then $I(\ell)=I_{0}(\ell)$ because our generalized random typing process produces words independently from the previous ones. If the context are characters, then $I(\ell)=I_{0}(\ell)$ is also warranted when $\ell_{0}=0$ because this is the case where self-repulsion of the space is suppressed. When $\ell_{0}>0$, (6) indicates that $I(\ell)$ cannot exceed $I_{0}(\ell)$.

In order to calculate the probability of producing a concrete word $w=$ $s_{1}, \ldots, s_{i}, \ldots, s_{\ell}$, where $s_{i}$ is the $i$-th character from $\Sigma$ of $w$, we use the shorthand

$$
\mathcal{P}_{i, j}=\prod_{h=i}^{j} p_{\Sigma}\left(S=s_{h}\right)
$$

By the independence between characters (except for space self-repulsion at distances smaller than $\ell_{0}$ ), the probability that a random word $W$ that has length $\ell$ coincides with $w=s_{1}, \ldots, s_{i}, \ldots, s_{\ell}$ is

$$
\begin{aligned}
p(W=w,\|w\|=\ell) & =\left(\prod_{i=1}^{\ell_{0}} p_{\Sigma \backslash\left\{\sigma_{0}\right\}}\left(S=s_{i}\right)\right)\left(\prod_{i=\ell_{0}+1}^{\ell} p_{\Sigma}\left(S=s_{i}\right)\right) p_{0} \\
& =\frac{p_{0}}{\left(1-p_{0}\right)^{\ell_{0}}}\left(\prod_{i=1}^{\ell} p_{\Sigma}\left(S=s_{i}\right)\right) \\
& =\frac{p_{0}}{\left(1-p_{0}\right)^{\ell_{0}}} \mathcal{P}_{1, \ell}
\end{aligned}
$$

the probability that a word has length $\ell$ is

$$
p(\|w\|=\ell)=p_{0}\left(1-p_{0}\right)^{\ell-\ell_{0}}
$$

and the probability of a word $w$ given its length is therefore

$$
\begin{aligned}
p(W=w \mid\|w\|=\ell) & =\frac{p(W=w,\|w\|=\ell)}{p(\|w\|=\ell)} \\
& =\frac{1}{\left(1-p_{0}\right)^{\ell}} \mathcal{P}_{1, \ell} .
\end{aligned}
$$

Applying (7), the self-information of a word $w$ of length $\ell$ is

$$
-\ln p(W=w,\|w\|=\ell)=b-\sum_{i=1}^{\ell} \ln p_{\Sigma}\left(S=s_{i}\right),
$$

where $b$ is defined as

$$
b=\ln \frac{\left(1-p_{0}\right)^{\ell_{0}}}{p_{0}} .
$$

Combining (8) and (9) with the definition of $I_{0}(\ell)$ in (2), gives

$$
I_{0}(\ell)=\frac{1}{\left(1-p_{0}\right)^{\ell}} \sum_{s_{1}, \ldots, s_{\ell}} \mathcal{P}_{1, \ell}\left(b-\sum_{i=1}^{\ell} \ln p_{\Sigma}\left(S=s_{i}\right)\right) .
$$

Bearing in mind that

$$
\begin{aligned}
\sum_{s_{1}, \ldots, s_{\ell}} \mathcal{P}_{1, \ell} & =\sum_{s_{1} \in \Sigma \backslash\left\{\sigma_{0}\right\}} \ldots \sum_{s_{i} \in \Sigma \backslash\left\{\sigma_{0}\right\}} \ldots \sum_{s_{\ell} \in \Sigma \backslash\left\{\sigma_{0}\right\}} \mathcal{P}_{1, \ell} \\
& =\sum_{s_{1} \in \Sigma \backslash\left\{\sigma_{0}\right\}} \ldots \sum_{s_{i} \in \Sigma \backslash\left\{\sigma_{0}\right\}} \ldots \sum_{s_{\ell} \in \Sigma \backslash\left\{\sigma_{0}\right\}} \prod_{h=1}^{\ell} p_{\Sigma}\left(S=s_{h}\right)
\end{aligned}
$$


Information content versus word length in random typing

$$
\begin{aligned}
& =\sum_{s_{1} \in \Sigma \backslash\left\{\sigma_{0}\right\}} \ldots \sum_{s_{i} \in \Sigma \backslash\left\{\sigma_{0}\right\}} \ldots \mathcal{P}_{1, \ell-1} \sum_{s_{\ell} \in \Sigma \backslash\left\{\sigma_{0}\right\}} p_{\Sigma}\left(S=s_{\ell}\right) \\
& =\left(1-p_{0}\right) \sum_{s_{1} \in \Sigma \backslash\left\{\sigma_{0}\right\}} \ldots \sum_{s_{i} \in \Sigma \backslash\left\{\sigma_{0}\right\}} \ldots \sum_{s_{\ell-1} \in \Sigma \backslash\left\{\sigma_{0}\right\}} \mathcal{P}_{1, \ell-1} \\
& =\left(1-p_{0}\right)^{2} \sum_{s_{1} \in \Sigma \backslash\left\{\sigma_{0}\right\}} \ldots \sum_{s_{i} \in \Sigma \backslash\left\{\sigma_{0}\right\}} \ldots \sum_{s_{\ell-2} \in \Sigma \backslash\left\{\sigma_{0}\right\}} \mathcal{P}_{1, \ell-2} \\
& =\ldots \\
& =\left(1-p_{0}\right)^{\ell},
\end{aligned}
$$

one can write

$$
I_{0}(\ell)=b+\frac{1}{\left(1-p_{0}\right)^{\ell}} \sum_{s_{1}, \ldots, s_{\ell}} \mathcal{P}_{1, \ell}\left(-\sum_{i=1}^{\ell} \ln p_{\Sigma}\left(S=s_{i}\right)\right) .
$$

Notice that

$$
\begin{aligned}
& \sum_{s_{1}, \ldots, s_{\ell}} \mathcal{P}_{1, \ell}\left(-\ln p_{\Sigma}\left(S=s_{i}\right)\right. \\
& \sum_{s_{1}, \ldots, s_{j-1}, s_{j+1}, \ldots, s_{\ell}}\left[\mathcal{P}_{1, j-1} \mathcal{P}_{j+1, \ell} \sum_{s_{j} \in \Sigma \backslash\left\{\sigma_{0}\right\}}-p_{\Sigma}\left(S=s_{j}\right) \ln p_{\Sigma}\left(S=s_{j}\right)\right]= \\
& \left(H_{\Sigma}(S)+p_{0} \ln p_{0}\right) \sum_{s_{1}, \ldots, s_{j-1}, s_{j+1}, \ldots, s_{\ell}} \mathcal{P}_{1, j-1} \mathcal{P}_{j+1, \ell} \\
& \left(H_{\Sigma}(S)+p_{0} \ln p_{0}\right)\left(1-p_{0}\right)^{\ell-1},
\end{aligned}
$$

where

$$
\begin{aligned}
H_{\Sigma}(S) & =-\sum_{s \in \Sigma} p_{\Sigma}(S=s) \ln p_{\Sigma}(S=s) \\
& =-\sum_{s \in \Sigma \backslash\left\{\sigma_{0}\right\}} p_{\Sigma}(S=s) \ln p_{\Sigma}(S=s)-p_{0} \ln p_{0}
\end{aligned}
$$

is the character entropy after the space-free prefix of length $\ell_{0}$. Therefore, applying (12) to (11) one finally obtains $I_{0}(\ell)=a \ell+b$, where

$$
a=\frac{1}{1-p_{0}}\left(H_{\Sigma}(S)+p_{0} \ln p_{0}\right)
$$

and $b$ is defined as in (10). Notice that the slope $a$ is always positive because $H_{\Sigma}(S) \geq 0$ as any entropy and, according to (13), $H_{\Sigma}(S)>p_{0} \ln p_{0}$ provided that $\lambda>1$ (recall that no character from $\Sigma$ has probability zero of occurring after the free-space prefix). Therefore, $I_{0}(\ell)$ grows linearly with $\ell$ for $\lambda>1$.

Table 1 summarizes the parameters of the linear relationship between $I_{0}(\ell)$ for our generalized random typing process and two particular cases: (a) equal letter probabilities (all characters except the space must be equally likely) [14] and (b) equal character probabilities (all characters including the space are equally likely) and empty words are allowed, i.e. $\ell_{0}=0$ [9]. Notice that (b) is a particular case of (a). Variant (a) [14] means that

$$
p_{\Sigma}(S=s)=\left\{\begin{array}{cc}
\frac{1-p_{0}}{\lambda-1} & \text { if } s \neq \sigma_{0} \\
p_{0} & \text { if } s=\sigma_{0},
\end{array}\right.
$$


Table 1. Summary of the linear dependency between the self-information content as a function of word length, $I_{0}(\ell)=a+b$, and related quantities for three major variants of the random typing process. $H_{\Sigma}(S)$ is the entropy of characters after the free-space prefix of length $\ell_{0}, p_{0}$ is the probability of space and $\lambda$ is the cardinality of $\Sigma . p_{s}$ is used as a shorthand for $p_{\Sigma}(S=s)$.

\begin{tabular}{|c|c|c|c|}
\hline \multicolumn{4}{|c|}{ Random typing } \\
\hline & Generalized & $\begin{array}{r}\text { Equal letter } \\
\text { probabilities } 14\end{array}$ & $\begin{array}{r}\text { Equal character } \\
\text { probabilities } \\
\text { (with } \ell_{0}=0[9] \text { ) }\end{array}$ \\
\hline$a$ & $\frac{1}{1-p_{0}}\left(H_{\Sigma}(S)+p_{0} \ln p_{0}\right)$ & $\ln \frac{\lambda-1}{1-p_{0}}$ & $\ln \lambda$ \\
\hline$b$ & $\ln \frac{\left(1-p_{0}\right)^{\ell_{0}}}{p_{0}}$ & $\ln \frac{\left(1-p_{0}\right)^{\ell_{0}}}{p_{0}}$ & $\ln \lambda$ \\
\hline$H_{\Sigma}(S)$ & $-\sum_{s \in \Sigma \backslash\left\{\sigma_{0}\right\}} p_{s} \ln p_{s}$ & $\left(1-p_{0}\right) \ln \frac{\lambda-1}{1-p_{0}}$ & $\ln \lambda$ \\
\hline & $-p_{0} \ln p_{0}$ & $-p_{0} \ln p_{0}$ & \\
\hline$p_{0}$ & $p_{0}$ & $p_{0}$ & $\frac{1}{\lambda}$ \\
\hline$p(W=w,\|w\|=\ell)$ & $\frac{p_{0}}{\left(1-p_{0}\right)^{\ell}} \mathcal{P}_{1, \ell}$ & $\frac{\left(1-p_{0}\right)^{\left(\ell-\ell_{0}\right)} p_{0}}{(\lambda-1)^{\ell}}$ & $\frac{1}{\lambda}$ \\
\hline$p(W=w \mid\|w\|=\ell)$ & $\frac{1}{\left(1-p_{0}\right)^{\ell}} \mathcal{P}_{1, \ell}$ & $\frac{1}{(\lambda-1)^{\ell}}$ & $\frac{1}{(\lambda-1)^{\ell}}$ \\
\hline
\end{tabular}

and is defined only by three parameters: $\ell_{0}, \lambda$ and $p_{0}$. The random typing process defined in [6] is a particular case with $\ell_{0}=0$. In a random typing process with equal letter probabilities, the character entropy after the space-free prefix is

$$
\begin{aligned}
H_{\Sigma}(S) & =(\lambda-1)\left(-\frac{1-p_{0}}{\lambda-1} \ln \frac{1-p_{0}}{\lambda-1}\right)-p_{0} \ln p_{0} \\
& =\left(1-p_{0}\right) \ln \frac{\lambda-1}{1-p_{0}}-p_{0} \ln p_{0} .
\end{aligned}
$$

Variant (b), the simplest random typing that has ever been presented to our knowledge, is defined with only one parameter, i.e. $\lambda\left(\ell_{0}=0\right.$ and $p_{0}=1 / \lambda$ in that case). (b) is known as the fair die rolling experiment [9] (see [7] for a version with $\ell_{0}=1$ and $\left.p_{0}=1 / \lambda\right)$.

\section{Conclusion}

We have shown that $I(\ell)=a \ell+b$ does not imply that speakers have made optimal choices as argued in [4. Uniform information density or related hypotheses (e.g., [5]) are not at all necesary to account for the linear correlation between $I(\ell)$ and $\ell$ : typing at random yields the same dependency independently from context. Our main point is that a linear correlation between information content and word length may simply arise internally, from the units making a word (e.g., letters) and not necessarily from the interplay between words and their context as suggested in [4]. However, future research should investigate if the parameters of the linear relationship predicted by random typing coincide with those of real texts or if a linear relationship is sufficient to account for the actual dependency between $I(\ell)$ and $\ell$ in real languages as it is suggested by the long-range correlations in texts at the level of words [15] or letters [16, 17] and 
the differences between random typing and real language at the level of the distribution of word frequencies [14, 18] or word lengths [19].

\section{Acknowledgments}

This work was supported by the project OpenMT-2 (TIN2009-14675-C03) from the Spanish Ministy of Science and Innovation (RFC).

\section{References}

[1] G. K. Zipf. The psycho-biology of language. Houghton Mifflin, Boston, 1935.

[2] S. Semple, M. J. Hsu, and G. Agoramoorthy. Efficiency of coding in macaque vocal communication. Biology Letters, 6:469-471, 2010.

[3] R. Ferrer-i-Cancho and D. Lusseau. Efficient coding in dolphin surface behavioral patterns. Complexity, 14(5):23-25, 2009.

[4] S. T. Piantadosi, H. Tily, and E. Gibson. Word lengths are optimized for efficient communication. Proceedings of the National Academy of Sciences, 108(9):3526-3529, 2011.

[5] T. F. Jaeger. Redundancy and reduction: Speakers manage syntactic information density. Cognitive Psychology, 61(1):23 - 62, 2010.

[6] G. A. Miller and N. Chomsky. Finitary models of language users. In R. D. Luce, R. Bush, and E. Galanter, editors, Handbook of Mathematical Psychology, volume 2, pages 419-491. Wiley, New York, 1963.

[7] W. Li. Random texts exhibit Zipf's-law-like word frequency distribution. IEEE T. Inform. Theory, 38(6):1842-1845, 1992.

[8] U. Cohen Priva. Using information content to predict phone deletion. In Proceedings of the 27th West Coast Conference on Formal Linguistics, pages 90-98. Cascadilla Proceedings Project, Somerville, MA, 2008.

[9] R. Suzuki, P. L. Tyack, and J. Buck. The use of Zipf's law in animal communication analysis. Anim. Behav., 69:9-17, 2005.

[10] B. Conrad and M. Mitzenmacher. Power laws for monkeys typing randomly: the case of unequal probabilities. IEEE Transactions on Information Theory, 50(7):1403-1414, 2004.

[11] M. Prokopenko, N. Ay, O. Obst, and D. Polani. Phase transitions in least-effort communications. J. Stat. Mech., page P11025, 2010.

[12] R. Ferrer i Cancho. Zipf's law from a communicative phase transition. European Physical Journal B, 47:449-457, 2005.

[13] T. M. Cover and J. A. Thomas. Elements of information theory. Wiley, New York, 2006. 2nd edition.

[14] R. Ferrer-i-Cancho and R. Gavaldà. The frequency spectrum of finite samples from the intermittent silence process. Journal of the American Association for Information Science and Technology, 60(4):837-843, 2009.

[15] M. Montemurro and P. A. Pury. Long-range fractal correlations in literary corpora. Fractals, 10:451-461, 2002.

[16] W. Ebeling and T. Pöschel. Entropy and long-range correlations in literary English. Europhysics Letters, 26(4):241-246, 1994.

[17] F. Moscoso del Prado Martín. The universal 'shape' of human languages: spectral analysis beyond speech. PLoS ONE, page in press, 2011.

[18] R. Ferrer-i-Cancho and B. Elvevåg. Random texts do not exhibit the real Zipf's-law-like rank distribution. PLoS ONE, 5(4):e9411, 2009.

[19] D.-Y. Manin. Zipf's law and avoidance of excessive synonymy. Cognitive Science, 32(7):1075-1098, 2008. 